\title{
Effects of different cooking methods of oatmeal on preventing the diet-induced increase of cholesterol level in hypercholesterolemic rats
}

\author{
Yandong Ban ${ }^{1}$ Ju Qiu², Changzhong Ren ${ }^{3}$ and Zaigui Li ${ }^{1 *}$
}

\begin{abstract}
Background: The aim of present study is to investigate the influences of brewing and boiling on hypocholesterolemic effect of oatmeal in rats fed with a hypercholesterolemic diet.

Methods: Fifty-six male Sprague-Dawley rats were divided into 5 groups of 8 rats each with similar mean body weights and serum cholesterol concentrations. Rats were fed with the experimental diets containing $10 \%$ of oatmeal from two Chinese oat varieties which were brewed or boiled for 30 days. The lipids levels in serum, liver, and faeces were determined.

Results: The effects of feeding boiled oatmeal on lowering lipid concentrations in plasma and liver were more significant than that of brewed oatmeal $(P<0.05)$. Feeding boiled oatmeal was also more efficient in increasing fecal total lipids, cholesterol and bile acids as compared to feeding brewed oatmeal $(P<0.05)$. Boiled oatmeal had higher apparent viscosity and soluble $\beta$-glucan content than the brewed oatmeal did $(P<0.05)$.

Conclusion: These results indicated that the capability of boiled oatmeal in improving cholesterol metabolism is better than that of brewed oatmeal, which is mainly attributed to its higher soluble $\beta$-glucan content and apparent viscosity.
\end{abstract}

Keywords: Oatmeal, Brewing, Boiling, Hypercholesterolemia, Rats

\section{Introduction}

Dietary oat effectively lowers the plasma total cholesterol and low-density lipoprotein (LDL)-cholesterol concentrations and, hence, reduces the risk of cardiovascular disease in animal models and hypercholesterolemic subjects $[1,2]$. The health benefits of oat are mainly attributed to its high content of nutritional components, including $2.0 \sim 7.5 \%$ of $\beta$-glucan [3], 2 $\sim 12 \%$ of crude fat [4], $13 \sim 20 \%$ of protein [5] and around $60 \%$ of starch [6]. Previous studies have also shown that dietary full-fat from oat bran could promote excretions of fecal lipids and bile acids. Oat oil has high tocotrienols and sterols contents and, hence, hypocholesterolemic effect. As a result, oat bran with high lipids content lower serum total cholesterol and

\footnotetext{
* Correspondence: lizg@cau.edu.cn

'Laboratory of Cereal Science, College of Food Science and Nutritional

Engineering, China Agricultural University, Beijing 100083, China

Full list of author information is available at the end of the article
}

LDL-cholesterol concentrations [7] as well as the concentrations of rat plasma total cholesterol, LDL-cholesterol the cholesterol, free cholesterol, cholesterol ester and triglyceride levels of liver [8].

According to Wolever et al. [9], dietary oat $\beta$-glucan reduces plasma cholesterol total cholesterol and LDLcholesterol levels by increasing intestinal viscosity, which lowers the reabsorption of bile acids, leading to lower plasma lipids levels. Wood et al. [10] reported that there was a significant correlation between the peak levels of blood glucose concentration and the viscosity of $\beta$ glucan. Therefore, it is nutritionally favorable for $\beta$ glucan to have a high viscosity. On the other hand, as reported by Tong et al. [11], dietary hull-less barley $\beta$ glucan could reduce the concentration of plasma LDL cholesterol in hypercholesterolemic hamsters. However, 
this hypocholesterolemic effect was weaker than that of oat $\beta$-glucan because of the higher viscosity and prebiotic activity of oat $\beta$-glucan. Previous study demonstrated that the viscosity and hypocholesterolemic effect of $\beta$-glucan was positively correlated with its molecular weight, namely, the molecular weight of $\beta$-glucan may influence the hypocholesterolemic effect [12].

Thermal, physical and enzymatic treatments had significant effects on the water solubility and molecular weight of $\beta$-glucan and, thereby, could affect the viscosity of hull-less barley [13]. In addition to molecular weight of $\beta$-glucan, the soluble $\beta$-glucan content can also affect the lipids-lowering effect of oat or hull-less barley because soluble $\beta$-glucan can formhighly viscous solutions, which seems to be responsible for reducing the plasma cholesterol in humans and animals [14-16]. In China, brewing and boiling are the most common cooking methods for oatmeal. However, few publications can be found reporting the relationship between the cooking methods and the health impacts of oatmeal, especially the lipid-lowering activity.

The aim of this study was to investigate the effects of oatmeal cooking methods including brewing and boiling on cholesterol metabolism of Sprague-Dawley rats fed with two different varieties of oatmeal. The apparent viscosity, soluble $\beta$-glucan and other index of brewed and boiled samples were determined, in order to clarify the differences in their hypocholesterolemic abilities.

\section{Materials and methods Materials}

Oatmeals (Avena sativa L., Bayou NO.1 and Bayou NO.8) were provided by Beijing Lipid-lowering Oats Products Development Co. Ltd. Oatmeal Bayou NO.1 contained $12.9 \%$ protein, $75.9 \%$ total starch, $5.6 \%$ crude lipid and $5.6 \%$ crude fiber. Oatmeal Bayou NO. 8 contained $16.7 \%$ protein, $68.8 \%$ total starch, $5.5 \%$ crude lipid and $9.0 \%$ crude fiber. The compositions of oatmeals were determined using the methods of AACC-32-23 (2000), AOAC 996.11, GB/T55112008, GB/T5512-2008, GB6193-86, and GB/5009.32010, respectively.

\section{Oatmeal treatment}

To obtain brewed oatmeal sample, $250 \mathrm{~g}$ of oatmeal was mixed with $1 \mathrm{~L}$ of $100{ }^{\circ} \mathrm{C}$ hot deionized water, and then cooled at room temperature with constant stirring. Boiled oatmeal sample was prepared by cooking $250 \mathrm{~g}$ of oatmeal in $1 \mathrm{~L}$ of $100{ }^{\circ} \mathrm{C}$ hot deionized water with constant boiling for $5 \mathrm{~min}$ using an electronic cooker (Povos-PIB07,
Shanghai POVOS Electric Works Co. Ltd., Shanghai, China), and then cooling at room temperature.

\section{Apparent viscosity}

The heat treated oatmeal samples were filtrated through a 100 mesh filter cloth, respectively. The apparent viscosity of the filtrate was measured by a digital viscometer (Model NDJ-9S, Shanghai Precision and Scientific Instruments Co., Ltd., Shanghai, China) using a $3^{\#}$ rotor at a shear rate of $3 \mathrm{r} / \mathrm{min}$ and $25{ }^{\circ} \mathrm{C}$.

\section{Soluble $\beta$-glucan concentration}

The $\beta$-glucan concentration in the filtrate of treated oatmeal was determined by an enzymatic method using a mixed-linkage $\beta$-glucan assay kit (Megazyme International Ireland, Ltd., Wicklow, Ireland).

\section{Starch gelatinization}

The starch gelatinization was measured using an enzymatic method according to Xiong et al. [17] with some modifications. The treated oatmeal samples were freeze-dried, and then crushed into flours with a cyclone mill. The flour was passed through a 100 mesh sieve. The oatmeal flour (100 mg) was added to $15 \mathrm{~mL}$ buffer $(0.37 \%$ glacial acetic acid and $0.41 \%$ anhydrous sodium acetate in deionized water) and boiled for $1 \mathrm{~h}$ to get a full pasting sample. The full pasting sample was mixed with $1 \mathrm{~mL}$ of amyloglucosidase (enzyme activity $100 \mathrm{U} / \mathrm{mg}$ ) solutions $(25 \%)$ and incubated at $40{ }^{\circ} \mathrm{C}$ for $1 \mathrm{~h}$ in a test tube. The buffer mixed with enzyme solution and without oatmeal flour was used as a blank. After $1 \mathrm{~h}$ of incubation, $2 \mathrm{~mL}$ of $10 \% \mathrm{ZnSO}_{4} \cdot 7 \mathrm{H}_{2} \mathrm{O}, 1 \mathrm{~mL}$ of $0.5 \mathrm{~N} \mathrm{NaOH}$ and $7 \mathrm{~mL}$ of deionized water were added to the test tube. The reaction solution was filtered by filter paper (Whatman $40^{\#}$ ) The filtrate $(0.1 \mathrm{~mL})$ and copper reagent $\left(2 \mathrm{~mL}, 40 \mathrm{~g} \mathrm{NaCO}_{3}\right.$, $7.5 \mathrm{~g}$ tartaric acid, and $4.5 \mathrm{~g} \mathrm{CuSO}_{4} \cdot 5 \mathrm{H}_{2} \mathrm{O}$ in $1 \mathrm{~L}$ deionized water) were mixed and boiled for $6 \mathrm{~min}$. Then, the suspension in the tube was mixed with $2 \mathrm{~mL}$ molybdic acid solution (70 g molybdic acid, $10 \mathrm{~g}$ sodium tungstate, $40 \mathrm{~g} \mathrm{NaOH}$, and $250 \mathrm{~mL} \mathrm{H}_{3} \mathrm{PO}_{4}(85 \%)$ in $1 \mathrm{~L}$ deionized water), heated (temperature?) for $2 \mathrm{~min}$, and filled up (with what buffer) to $25 \mathrm{~mL}$. Absorbance at $420 \mathrm{~nm}$ of the solutions was measured using a spectrophotometer. Starch gelatinization was calculated using the following formula.

$$
\begin{aligned}
\text { Starch gelatinization }(\%)= & \left(\mathrm{A}_{\text {test sample }}-\mathrm{A}_{\text {blank }}\right) / \\
& \left(\mathrm{A}_{\text {full pasting sample }}-\mathrm{A}_{\text {blank }}\right) \\
& \times 100 \%
\end{aligned}
$$




\section{Protein dispersibility index}

Protein dispersibility index (PDI) was measured using a method described by Iwe et al. [18] with some modifications (). Briefly, the oatmeal flour (15 g) was blended in $250 \mathrm{~mL}$ of deionized water for $10 \mathrm{~min}$ in a XHF-D HI-speed dispersator (Ningbo Scientific Biotechnology, Ningbo, China) and then centrifuged at $5000 \mathrm{~g}$ at $25{ }^{\circ} \mathrm{C}$. The protein concentration of supernatant was determined by Kjeldahl method (AOAC 984.13). The PDI was calculated by the following formula.

$$
\begin{aligned}
\operatorname{PDI}(\%)= & \text { Solubility protein } / \text { Total protein } \\
& \text { content of sample } \times 100 \%
\end{aligned}
$$

\section{Animals and diets}

Fifty-six male Sprague-Dawley rats $(n=40)$ were obtained from College of Medicine, Xi'an JiaoTong University (Xi'an, China). All rats were housed individually in stainless steel cages under controlled temperature $\left(22 \pm 2{ }^{\circ} \mathrm{C}\right)$, humidity $(55 \pm 5 \%)$ and air flow conditions with a fixed $12 \mathrm{~h}$ light-dark cycle. After 1 week acclimation, rats were divided into 5 groups, namely, Control (C), Brewed Bayou No.1 (Br1), Boiled Bayou No.1 (Bo1), Brewed Bayou No.8 (Br8), and Boiled Bayou No.8 (Bo8). The average body weight of the rats was similar for each group. The rats in different groups were fed with different oatmeals and deionized water for 5 weeks. Experimental diets shown in Table 1 were prepared according to the American Institute of Nutrition (AIN)-93G formula. As shown in Table 1, the cholesterol, bile salts and lard was added to standard diet in order to elevate the concentration of serum cholesterol. Rat feces were collected for 3 days before sacrification. The rats were fasted for $16 \mathrm{~h}$ and then sacrificed by the removal of blood from the abdominal aorta. Their livers were quickly dissected and washed in ice-cold saline solution to remove the blood, and then stored at $-80{ }^{\circ} \mathrm{C}$ in a deep freezer for biochemical analysis.

This study was carried out according to the P.R. China legislation regarding the use and care of laboratory animals and was approved by the Animal Ethics Committee of Northwest A\&F University (Yangling, China).

\section{Analysis of metabolic parameters in rats}

The concentrations of plasma lipids were measured using an Automatic Chemistry Analyzer (7020, Hitachi, Tokyo, Japan) at Biochemical Clinical Laboratory, Yangling Demonstration Zone Hospital, Yangling, China. The liver lipids extracted by tissue lysate from
Table 1 Diet composition (g/1000 g diet)

\begin{tabular}{llllll}
\hline & $\mathrm{C}$ & $\mathrm{Br} 1$ & $\mathrm{Bo1}$ & $\mathrm{Br} 8$ & $\mathrm{Bo8}$ \\
\hline Casein & 200 & 187.1 & 187.1 & 183.3 & 183.3 \\
Corn Starch & 397 & 321.1 & 321.1 & 328.2 & 328.2 \\
Soybean oil & 70 & 64.4 & 64.4 & 64.5 & 64.5 \\
Cellulose & 50 & 44.4 & 44.4 & 41.0 & 41.0
\end{tabular}

Oatmeal

\begin{tabular}{llllll}
$\begin{array}{l}\text { Brewed Bayou No.1 } \\
\text { Boiled Bayou No.1 }\end{array}$ & \multicolumn{5}{c}{100} \\
Brewed Bayou No.8 & & & & 100 & \\
Boiled Bayou No.8 & & & & & 100 \\
Maltodextrin 10 & 132 & 132 & 132 & 132 & 132 \\
Sucrose & 38.486 & 38.486 & 38.486 & 38.486 & 38.486 \\
-Butylhydroquinone & 0.014 & 0.014 & 0.014 & 0.014 & 0.014 \\
Mineral Mix & 35 & 35 & 35 & 35 & 35 \\
Vitamin Mix & 10 & 10 & 10 & 10 & 10 \\
Choline Bitartrate & 2.5 & 2.5 & 2.5 & 2.5 & 2.5 \\
L-Cystine & 3 & 3 & 3 & 3 & 3 \\
Cholesterol & 10 & 10 & 10 & 10 & 10 \\
Bile salt & 2 & 2 & 2 & 2 & 2 \\
Lard & 50 & 50 & 50 & 50 & 50 \\
\hline
\end{tabular}

The diets were prepared according to the AIN-93G formula with some modifications as described in the section of Materials and methods. Control, C; Brewed Bayou No.1, Br1; Boiled Bayou No.1, Bo1; Brewed Bayou No.8, Br8; Boiled Bayou No.8, Bo8

tissue lipids assay kits were chemically measured using the method as the kits described. The concentrations of liver lipids were measured by a Tissue triglyceride assay kit, E1003-2; Tissue total cholesterol assay kit, E1015; and Tissue free cholesterol assay kit, E1016 (Applygen Technologies Co., Ltd, Beijing, China). Liver was made to be paraffin sections, and its structure was observed by H\&E staining method. The fecal total lipids contents were measured by Soxhlet method. The cholesterol and fecal bile acids contents were measured using the Tissue total cholesterol assay kit, E1015 (Applygen Technologies Co., Beijing, China) and Rat Bile Acid ELISA Kit (Nanjing Sen Shellfish Gamma Biotechnology Co., Ltd., Nanjing, China), respectively.

\section{Statistical analysis}

The data were expressed as the mean \pm standard errors (SE) and analyzed by SPSS (Version 12.0 for Windows, SPSS Inc., Chicago, IL, USA) using Tukey-Kramer's multiple comparison post hoc test and $t$-test. Statistical significance was defined as $P<$ 0.05 . 
Table 2 Effects of eating methods on the growth parameters of Sprague-Dawley rats fed with a hypercholesterolemic diet ${ }^{\mathrm{a}}$

\begin{tabular}{|c|c|c|c|c|c|}
\hline & $C$ & $\mathrm{Br} 1$ & Bo1 & $\mathrm{Br} 8$ & Bo8 \\
\hline \multicolumn{6}{|l|}{ Intake (g/day) } \\
\hline Food & $25.1 \pm 0.4$ & $25.2 \pm 0.5$ & $25.3 \pm 0.4$ & $25.0 \pm 0.3$ & $25.4 \pm 0.3$ \\
\hline Water & $19.8 \pm 0.4$ & $18.9 \pm 0.5$ & $19.0 \pm 0.5$ & $19.0 \pm 0.5$ & $19.8 \pm 0.4$ \\
\hline \multicolumn{6}{|l|}{ Weight (g) } \\
\hline Initial body weight & $281 \pm 5$ & $279 \pm 6$ & $277 \pm 4$ & $283 \pm 5$ & $279 \pm 7$ \\
\hline Final body weight & $327 \pm 9$ & $333 \pm 10$ & $332 \pm 9$ & $323 \pm 13$ & $323 \pm 10$ \\
\hline Body weight gain & $56 \pm 9$ & $54 \pm 8$ & $55 \pm 5$ & $50 \pm 7$ & $54 \pm 8$ \\
\hline Liver & $10.7 \pm 0.3$ & $10.9 \pm 0.8$ & $11.0 \pm 0.6$ & $10.9 \pm 0.5$ & $11.3 \pm 0.2$ \\
\hline
\end{tabular}

${ }^{a}$ Means and standard errors (SE) were determined from 8 rats per group. Control, C; Brewed Bayou No.1, Br1; Boiled Bayou No.1, Bo1; Brewed Bayou No.8, Br8; Boiled Bayou No.8, Bo8

\section{Results}

\section{Growth parameters of rats}

The growth parameters of rats were not affected by feeding different oatmeals. The initial body weight, final body weight, body weight gain, food intake, water intake and liver weight did not differ from each other significantly among the five groups (Table 2).

\section{Plasma lipids}

Both boiled and brewed oatmeal prevented the dietinduced increase of the concentrations of plasma total cholesterol, LDL-cholesterol and triglycerides as compared to the control group $(P<0.05)$ (Fig. 1a, b, d). The capabilities of boiled oatmeal, both Bo1 and Bo8, in lowering plasma total cholesterol, LDL-cholesterol and
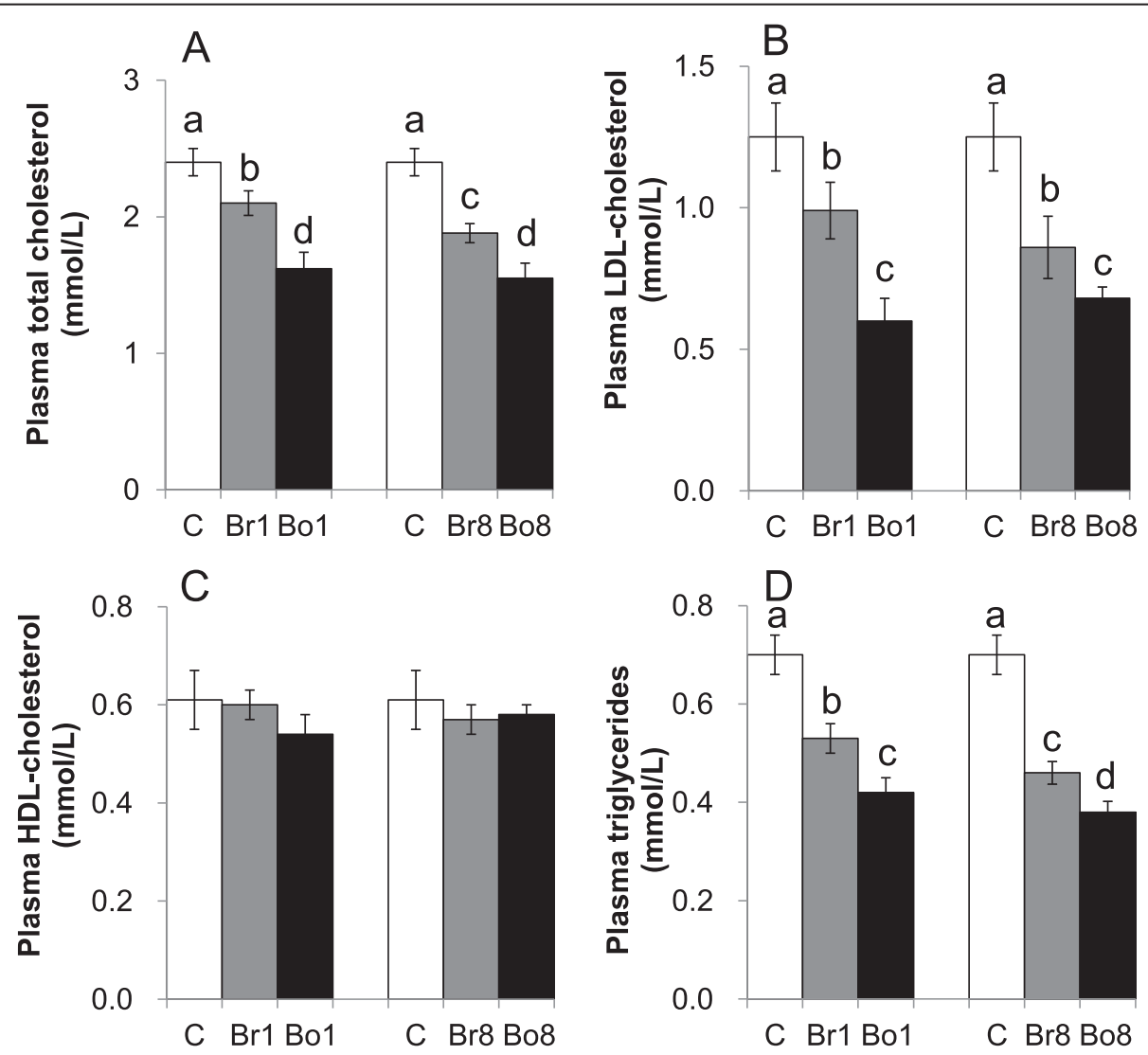

Fig. 1 Plasma total cholesterol (a), LDL-cholesterol (b), HDL-cholesterol (c), and triglycerides (d) of the experimental rats. Means and standard errors were determined from 8 rats per group. Different superscript letters indicate significant differences at $P<0.05$ 
triglycerides concentrations were more significant than those of brewed oatmeal $(P<0.05)$ (Fig. 1a, b, d). Moreover, the Br8 prevented the diet-induced increase of the plasma total cholesterol concentration more significantly than the $\mathrm{Br} 1 \quad(P<0.05)$ (Fig. 1a). The plasma triglycerides concentrations in both $\mathrm{Br} 8$ and Bo8 were significantly lower than those in both Br1 and Bo1 $(P<0.05)$ (Fig. 1d). There was no difference in the concentrations of plasma HDL-cholesterol among the five groups (Fig. 1c).

\section{Liver lipids}

As shown in Fig. 2, the concentrations of liver cholesterol, free cholesterol, cholesterol ester and triglycerides in Brewing Bayou NO.1 (Br1), Boiling Bayou NO.1 (Bo1), Brewing Bayou NO.8 (Br8) and Boiling Bayou NO.8 (Bo8) groups were significantly lower than those in the control group $(P<0.05)$. Moreover, the effect of boiled groups in lowering liver lipid concentrations was stronger than that of brewed groups $(P<0.05)$. H\&E stained liver structure was showed in Fig. 3. These liver lipids concentrations were not significantly different between two varieties.

\section{Fecal lipids}

The fecal total lipids excretion, cholesterol and bile acids excretion were increased in oatmeal groups compared with the control group $(P<0.05)$ (Table 3$)$. There was no difference in fecal weight among the 5 groups. The capabilities of boiled oatmeal, both two varieties, to increase fecal total lipids excretion, cholesterol and bile acids were stronger than that of brewed oatmeal $(P<$ 0.05). The ability of Br8 to increase bile acids was stronger than that of $\operatorname{Br} 1(P<0.05)$. In addition, the ability of Bo8 to increase fecal total lipids excretion, cholesterol and bile acids was stronger than that of Bo1 $(P<0.05)$.

\section{Chemical and physical characteristics of oatmeal}

The brewing and boiling methods had different effects onthe apparent viscosity, soluble $\beta$-glucan concentration, starch gelatinization and PDI of oatmeal (Fig. 4). The boiled oatmeal had higher apparent viscosity, soluble $\beta$-glucan content, gelatinization index and PDI $(P<0.05)$ than the brewed one. The apparent viscosity, soluble $\beta$-glucan content and PDI of Bo8 and Br8 were significantly higher than those of Bo1 and Br1 $(P<0.05)$ (Fig. 4a, b, d).
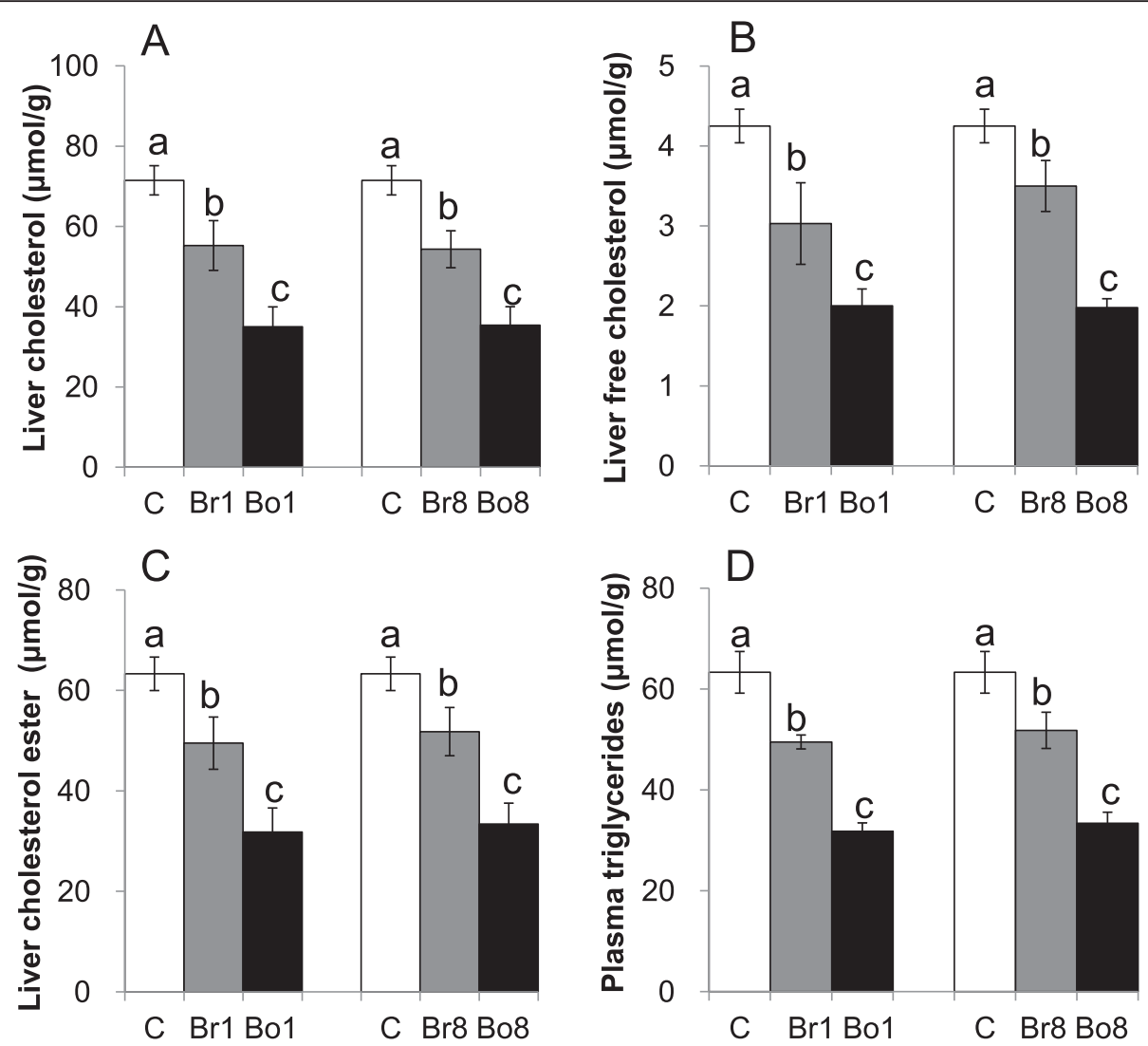

Fig. 2 Liver total cholesterol (a), free cholesterol (b), cholesterol ester (c), and triglycerides (d) of the experimental rats. Means and standard errors were determined from 9 rats per group. Different superscript letters indicate significant differences at $P<0.05$ 

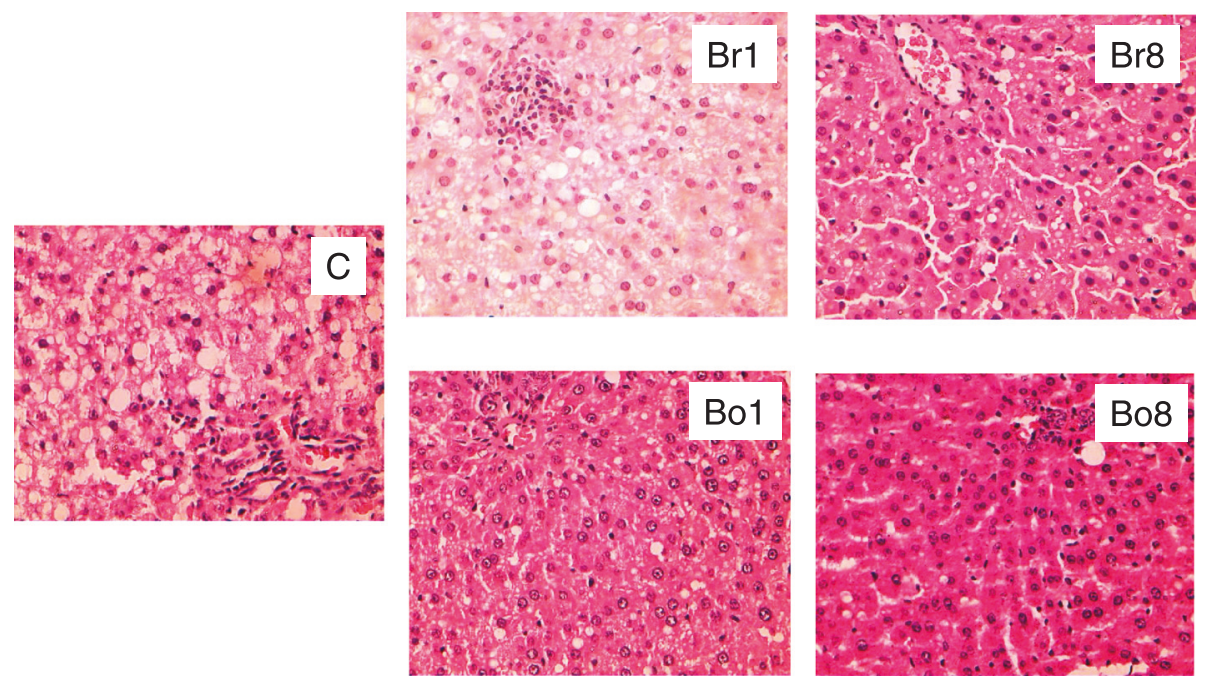

Fig. 3 Liver structure of the experimental rats (H\&E stain; 200X)

\section{Discussion}

Numerous studies have revealed the cholesterol metabolism of oats in animal models and hypercholesterolemic subjects may play the beneficial effects, and thereby reduce the risk of cerebrovascular disease $[1,2,19,20]$. So far, a large number of studies have focused on the effects of oat functional ingredients and its mechanism, but the effect of cooking method of oatmeal on cholesterol-lowering activity is still unknown. In present study, boiling methods has been demonstrated to be a more efficacy cooking method for oatmeal for improving its effects in lowering plasma lipid concentration. Boiled oats (both two varieties) lowered the concentrations of plasma total cholesterol, LDL-cholesterol and triglycerides more significantly than the brewed oatmeal. In addition, the ability of boiled oatmeal to lower liver lipids concentrations was stronger than that of brewed oatmeal $(P<0.05)$. The findings were new because the effects of cooking methods on oatmeal cholesterol-lowering activity was not reported much elsewhere. We also found that Bayou No.8 had better lowering effect of plasma lipids than Bayou
No.1. It was in agreement with the reports that the cholesterol-lowering effect was attributed to its $\beta$ glucan content $[9,21]$.

It has been widely reported that dietary oats reduces the plasma lipids concentrations by promoting the excretion of fecal total lipids and bile acids, and regulating the activities of 3-hydroxy-3-methyl glutarylcoenzyme A reductase and cholesterol 7- $\alpha$ hydroxylase CYP7A1 [22]. In present study, the fecal total lipids, cholesterol and bile acids excretion were increased in all of oatmeal groups compared with the control group $(P<0.05)$. The capability of boiled oatmeal to increase fecal lipids excretion was stronger than that of brewed oatmeal $(P<0.05)$. The results indicate that the boiling method contributes to decrease plasma and liver cholesterol concentrations by strengthening the inhibition of cholesterol absorption in intestine through high intestinal viscosity provided by high soluble $\beta$-glucan content.

Studies have shown that the highly water-soluble $\beta$-glucan plays the better role in lowering cholesterol than $\beta$-glucan with the low water-solubility $[10,12]$. The soluble $\beta$-glucan shows the capacity of forming

Table 3 Effects of eating methods on the fecal parameters of Sprague-Dawley rats fed with a hypercholesterolemic diet ${ }^{1}$

\begin{tabular}{|c|c|c|c|c|c|}
\hline & C & $\mathrm{Br} 1$ & Bo1 & $\mathrm{Br} 8$ & Bo8 \\
\hline Fecal weight (g/day) & $2.50 \pm 0.21$ & $2.55 \pm 0.23$ & $2.61 \pm 0.17$ & $2.58 \pm 0.19$ & $2.66 \pm 0.11$ \\
\hline \multicolumn{6}{|l|}{ Fecal lipids (mg/day) } \\
\hline Total lipids & $91 \pm 9^{a}$ & $132 \pm 11^{b}$ & $197 \pm 10^{c}$ & $136 \pm 8^{b}$ & $241 \pm 22^{d}$ \\
\hline Cholesterol & $44 \pm 5^{a}$ & $59 \pm 6^{\mathrm{b}}$ & $90 \pm 8^{c}$ & $64 \pm 7^{b}$ & $117 \pm 13^{d}$ \\
\hline Bile acids & $57 \pm 3^{\mathrm{a}}$ & $67 \pm 5^{b}$ & $93 \pm 4^{c}$ & $89 \pm 5^{c}$ & $121 \pm 11^{d}$ \\
\hline
\end{tabular}

${ }^{1}$ Means and standard errors (SE) were determined from 8 rats per group. Different superscript letters indicated significant differences at $P<0.05$ (Tukey-Kramer's multiple comparison post hoc test). Control, C; Brewed Bayou No.1, Br1; Boiled Bayou No.1, Bo1; Brewed Bayou No.8, Br8; Boiled Bayou No.8, Bo8 

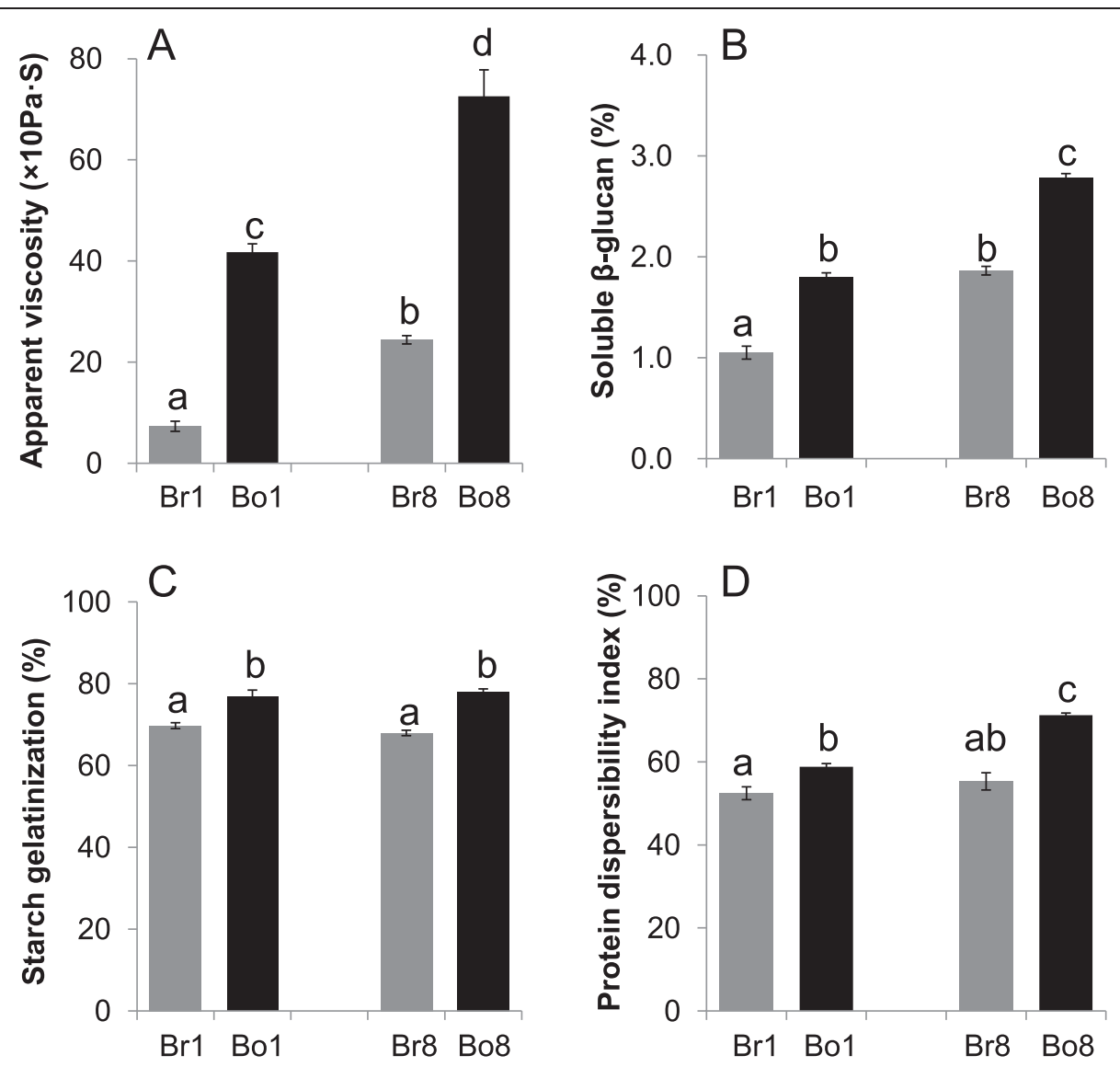

Fig. 4 Apparent viscosity (a), soluble $\beta$-glucan (b), starch gelatinization (c), and protein dispersibility index (d) of the brewed oatmeal and boiled oatmeal. Means and standard errors were determined in triplicate for each sample. Different superscript letters indicate significant differences at $P<0.05$

highly viscous solutions which seems to be responsible for the reduction of plasma cholesterol by increasing intestinal viscosity [14-16]. In addition, the soluble $\beta$-glucan is fermented by the intestinal microflora to produce the short chain fatty acids (SCFAs) which also results in the decrease of cholesterol [23]. Bell et al. [24] reported that these SCFAs, absorbed through the portal vein, improved cholesterol metabolism by increasing catabolism of LDLcholesterol or regulating the related enzymes activities. The boiled oatmeal had higher soluble $\beta$-glucan content (Fig. 4a) and apparent viscosity (Fig. 4b) than the brewed oatmeal $(P<0.05)$, which might be related to the higher efficacy of boiled oatmeal in lowering lipids. Our study was in agree with Izydorczyk et al. [13] which involved in hull-less barley. The boiled oatmeal showed higher starch gelatinization and PDI compared with the brewed oatmeal $(P<0.05)$ (Fig. 4c, d). Because the boiling applied more thermal energy which results in the increase of starch gelatinization and PDI, boiled oatmeal has a loose organizational structure. This may be the reason why boiling increases soluble $\beta$-glucan content of oatmeal, thus showing better lipid-lowering effectiveness than brewing.

\section{Conclusions}

The present study clearly revealed that cooked oatmeal can lower plasma and liver lipids concentrations in Sprague-Dawley rats fed with a hypercholesterolemic diet, and boiled oatmeal is more effective than the brewed one. The boiled oatmeal improved cholesterol metabolism by enhancing the excretions of fecal total lipids, cholesterol and bile acids. Furthermore, the better hypocholesterolemic effect of boiled oatmeal was proven to be positively correlated to its higher soluble $\beta$-glucan content and apparent viscosity as compared to the brewed oatmeal.

\section{Competing interests}

None of the authors had any conflict of interest at the time the research was done. 


\section{Authors' contributions}

YB, Conception and design, Analysis and interpretation, Data Collection,Writing the article, Critical revision of the article, Statistical analysis and Overall Responsibility. JQ, Conception anddesign, Analysis and interpretation, Data Collection, Writing the article, Critical revision of the article, Statisticalanalysis and Overall Responsibility. CR, Analysis and interpretation, Data Collection and Overall Responsibility. ZL, Conception and design, Analysis and interpretation, Data Collection, Writing the article, Critical revision of thearticle, Statistical analysis and Overall Responsibility. All authors read and approved the finalmanuscript.

\section{Acknowledgments}

This research was funded by the Chinese Agricultural Research System (grant number: CARS-08-D-3). We appreciate Beijing Lipid-lowering Oats Products Development Co. Ltd for their kindy support to this work.

\section{Author details}

'Laboratory of Cereal Science, College of Food Science and Nutritional Engineering, China Agricultural University, Beijing 100083, China. ${ }^{2}$ Institute of Food and Nutrition Development, Ministry of Agriculture, 12 Zhongguancun South Street, Haidian District, Beijing 100081, China. ${ }^{3}$ Oat Engineering Centre, Baicheng Academy of Agricultural Sciences, Baicheng, Jilin 137000, China.

Received: 22 September 2015 Accepted: 16 October 2015

Published online: 24 October 2015

\section{References}

1. Chen $\mathrm{CW}$, Cheng HH. A rice bran oil diet increases LDL-receptor and HMG-CoA reductase mRNA expressions and insulin sensitivity in rats. J Nutr. 2006;136:1472-6

2. Queenan KM, Stewart ML, Smith KN, Thomas W, Fulcher RG, Slavin JL. Concentrated oat beta-glucan, a fermentable fiber, lowers serum cholesterol in hypercholesterolemic adults in a randomized controlled trial. Nutr J. 2007;6:6.

3. Andersson AAM, Börjesdotter D. Effects of environment and variety on content and molecular weight of $\beta$-glucan in oats. J Cereal Sci. 2011:54:122-8.

4. Aro H, Järvenpää E, Könkö K, Hietaniemi V. The characterization of oat lipids produced by supercritical fluid technologies. J Cereal Sci. 2007:45:116-9.

5. Mirmoghtadaie L, Kadivar M, Shahedi M. Effects of succinylation and deamidation on functional properties of oat protein isolate. Food Chem. 2009;114:127-31.

6. Hoover R, Smith C, Zhou Y, Ratnayake RM. Physicochemical properties of Canadian oat starches. Carbohyd Polym. 2003;52:253-61.

7. Gerhardt AL, Gallo NB. Full-fat rice bran and oat bran similarly reduce hypercholesterolemia in humans. J Nutr. 1998;128:865-9.

8. Tong LT, Zhong K, Liu L, Guo L, Cao L, Zhou S. Oat oil lowers the plasma and liver cholesterol concentrations by promoting the excretion of faecal lipids in hypercholesterolemic rats. Food Chem. 2014;142:129-34.

9. Wolever TMS, Tosh SM, Gibbs AL, Brand-Miller J, Duncan AM, Hart V, et al. Physicochemical properties of oat $\beta$-glucan influence its ability to reduce serum LDL cholesterol in humans: a randomized clinical trial. Am J Clin Nutr. 2010;92:723-32

10. Wood PJ, Beer MU, Butler G. Evaluation of role of concentration and molecular weight of oat $\beta$-glucan in determining effect of viscosity on plasma glucose and insulin following an oral glucose load. Brit J Nutr. 2000;84:19-23.

11. Tong LT, Zhong K, Liu L, Zhou X, Qiu J, Zhou S. Effects of dietary hull-less barley b-glucan on the cholesterol metabolism of hypercholesterolemic hamsters. Food Chem. 2015;169:344-9.

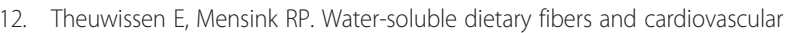
disease. Physiol Behav. 2008;94:285-92.

13. Izydorczyk MS, Storsley J, Labossiere D, MacGregor AW, Rossnagel BG. Variation in total and soluble $\beta$-glucan content in hulless barley: effects of thermal, physical, and enzymic treatments. J Agric Food Chem. 2000:48:982-9.

14. Jenkins DJ, Jenkins AL, Wolever TM, Vuksan V, Rao AV, Thompson LU, et al. Effect of reduced rate of carbohydrate absorption on carbohydrate and lipid metabolism. Eur J Clin Nutr. 1995;49:S68-73.

15. Kahlon TS, Chow Fl. Hypocholesterolemic effects of oat, rice, and barley dietary fibers and fractions. Cereal Foods World. 1997;42:86-92.
16. Yokoyama WH, Hudson CA, Knuckles BE, Chiu MCM, Sayre RN, Turnlund JR, et al. Effect of barley $\beta$-glucan in durum wheat pasta on human glycemic response. Cereal Chem. 1997;74:293-6.

17. Xiong Y, Bartle SJ, Preston RL. Improved enzymatic method to measure processing effects and starch availability in sorghum grain. J Anim Sci. 1990:68:3861-70

18. Iwe MO, Van Zuilichem DJ, Ngoddy PO, Lammers W. Amino acid and protein dispersibility index (PDI) of mixtures of extruded soy and sweet potato flours. LWT-Food Sci Technol. 2001;34:71-5.

19. Berg A, König D, Deibert P, Grathwohl D, Berg A, Baumstark MW, et al. Effect of an oat bran enriched diet on the atherogenic lipid profile in patients with an increased coronary heart disease risk. Ann Nutr Metab. 2003:47:306-11.

20. Ryan D, Kendall M, Robards K. Bioactivity of oats as it relates to cardiovascular disease. Nutr Res Rev. 2007;20:147-62.

21. Tiwari $U$, Cummins E. Dietary exposure assessment of $\beta$-glucan in a barley and oat based bread. LWT-Food Sci Technol. 2010;47:413-20.

22. Tong LT, Zhong K, Liu L, Qiu J, Guo L, Zhou X, et al. Effects of dietary wheat bran arabinoxylans on cholesterolmetabolism of hypercholesterolemic hamsters. Carbohyd Polym. 2014;112:1-5.

23. Drzikova B, Dongowski G, Gebhardt E, Habel A. The composition of dietary fibre-rich extrudates from oat affects bile acid binding and fermentation in vitro. Food Chem. 2005;90:181-92.

24. Bell S, Goldman VM, Bistrian BR, Arnold AH, Ostroff G, Forse RA. Effect of $\beta$-glucan from oats and yeast on serum lipids. Crit Rev Food Sci Nutr. 1999;39:189-202.

\section{Submit your next manuscript to BioMed Central and take full advantage of:}

- Convenient online submission

- Thorough peer review

- No space constraints or color figure charges

- Immediate publication on acceptance

- Inclusion in PubMed, CAS, Scopus and Google Scholar

- Research which is freely available for redistribution 